\title{
Atlasing white matter and grey matter joint \\ contributions to resting-state networks in the
}

\section{human brain}

\author{
Victor Nozais ${ }^{1,2,3,4}$ Stephanie J Forkel ${ }^{1,2,3,4,5}$ Laurent Petit ${ }^{1,2,3}$ \\ Michel Thiebaut de Schotten ${ }^{1,2,3,4}$ Marc Joliot $^{1,2,3}$
}

1 UMR 5293, GIN, IMN, Univ. Bordeaux, Bordeaux, France, 2 UMR5293, GIN, IMN, CNRS, Bordeaux, France, 3 UMR 5293, GIN, IMN, CEA, Bordeaux, France

4 Brain Connectivity and Behaviour Laboratory, Sorbonne Universities, Paris, France

5 Centre for Neuroimaging Sciences, Department of Neuroimaging, Institute of Psychiatry, Psychology and Neuroscience, King's College London, London, UK

\section{Abstract}

Over the past two decades, the study of resting-state functional magnetic resonance imaging ( $f M R I)$ has revealed the existence of multiple brain areas displaying synchronous functional blood oxygen level-dependent signals (BOLD) - resting-state networks (RSNs). The variation in functional connectivity between the different areas of a restingstate network or between multiple networks, have been extensively studied and linked to cognitive states and pathologies. However, the white matter connections supporting each network remain only partially described. In this work, we developed a data-driven method to systematically map the white and grey matter contributing to resting-state networks. Using the Human Connectome Project, we generated an atlas of 30 restingstate networks, each with two maps: white matter and grey matter. By integrating structural and functional neuroimaging data, this method builds an atlas that unlocks the joint anatomical exploration of white and grey matter to resting-state networks. The method also allows highlighting the overlap between networks, which revealed that most (89\%) of the brain's white matter is shared amongst multiple networks, with $16 \%$ shared by at least 7 resting-state networks. These overlaps, especially the existence of regions shared by numerous networks, suggest that white matter lesions in these areas might strongly impact the correlations and the communication within resting-state networks. 
We provide an open-source software to explore the joint contribution of white and grey matter to RSNs and facilitate the study of the impact of white matter damage on RSNs.

\section{Introduction}

Since the early 1990s, functional magnetic resonance imaging (fMRI) peers inside the workings of the living human brain (1). Task fMRI unveiled countless aspects of brain functioning in healthy participants and patients. However, paradigm-free resting-state fMRI (rs-fMRI) analysis shows a striking correspondence with tasks-related fMRI (2) yet provides the most comprehensive depiction of the functional organisation of the brain. Rs-fMRI explores the awake brain at rest, when no specific external task is required from the participant during which quasi-periodic low-frequency oscillations in the fMRI signal - blood-oxygen-level-dependent signal or BOLD - spontaneously occur (3). Distant brain regions display synchronous BOLD signal oscillations, testifying to functional connectivity between regions and forming intrinsic functional networks, so called resting-state networks (RSNs) (4-6). RSNs are related to cognition (2), and their alteration has been linked to various brain pathologies (7-9), potentially opening up this field to a wide range of applications, including in the clinical realm (10). Hence, resting-state acquisition is appealing and much less demanding than the active participant involvement in a task.

The identification of RSNs has been tackled in multiple ways (11). One of the most popular approaches is an independent component analysis (ICA) $(5,12,13)$, a data-driven method of signal separation (14) able to identify and extract independent components (ICs) corresponding to RSNs in the resting-state signal across the brain. From such components, resting-state networks and their grey matter maps can be identified.

With the progress of the functional connectivity framework, the question of the underlying structural connectivity became pressing. Indeed, understanding the anatomical drivers of the functional connection between multiple regions is necessary to properly study the dynamics and biological relevance of these networks. In that regard, the advent of diffusion-weighted imaging (DWI) tractography enabled the description of white matter circuits in the living human brain. DWI measures the preferential orientations of water diffusion in the brain (15), which mostly follow axonal directions. Using this information, tractography algorithms piece together local estimates of water 
diffusion to reconstruct white matter pathways (16). DWI is the most powerful noninvasive in-vivo tool for mapping the white matter anatomy (17) and estimating structural connectivity between brain regions $(18,19)$. Leveraging tractography, the joint study of functional and structural connectivity has become an active field of research. However, most works focused on the comparison of functional connectivity and structural connectivity between pairs of grey matter brain parcels, $(20,21)$ or provided white matter maps related to resting-state networks either focused on a single network (22-25) or a restricted number of RSNs (26-28) with limited statistical confirmation of structuralfunctional connectivity relationships (22-25). Of note, ICA applied to white matter tractography data produces circuits whose grey matter projections resemble restingstate networks $(29,30)$. These results demonstrate that information about the organisation of RSNs can also be extracted from white matter data and might be complementary to the information provided by resting-state BOLD signal analysis. However, to the best of our knowledge, a comprehensive description of the white matter circuits in all identifiable resting-state networks is still lacking.

Such endeavour could be achieved, in principle, by using the Functionnectome $(30,31)$, a recently developed method that combines fMRI with tractography by projecting the grey matter BOLD signal onto white matter pathways.

In the present study, we extended our previous approach - the Functionnectome methodology (30) - to RSNs, integrating the grey matter resting-state signal with white matter connections, and analysed the resulting data through ICA. In so doing, we produced the most comprehensive atlas of 30 RSNs specifying their grey matter maps together with their white matter circuitry - the WhiteRest atlas. This atlas unlocks the systematic exploration of white matter components supporting resting-state networks. The atlas comes with companion software, the WhiteRest tool, a module of the Functionnectome that will facilitate this exploration and assist the investigation of brain lesions' effects on RSNs and cognition. 


\section{Results}

Mapping the resting brain: RSNs in white matter and grey matters

Rs-fMRI scans derived from the Human Connectome Project (32) were converted into functionnectome volumes using the Functionnectome software $(30,31)$ (available at http://toolkit.bcblab.com). The original rs-fMRI and functionnectome volumes were simultaneously entered into an Independent Component Analysis for each participant. The resulting individual independent components were then automatically classified using MICCA (33), generating 30 IC groups, each group corresponding to one restingstate network. These groups were used to create RSN z-maps with paired white matter and grey matter maps (Fig. 1) - the WhiteRest atlas. 


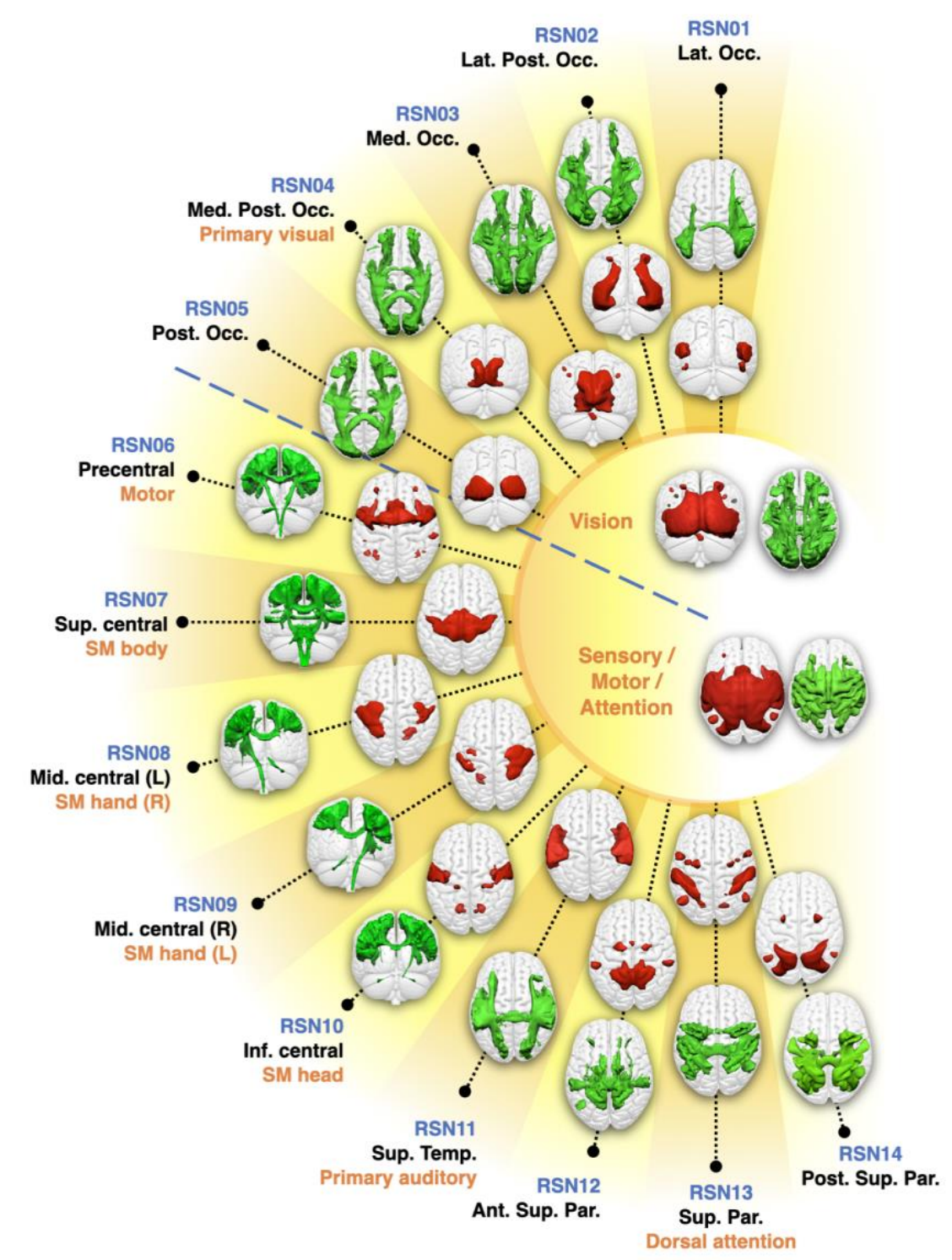

Figure 1: WhiteRest resting-state atlas of the visual and sensory/motor/attention domains.

This composite figure shows the white matter 3D maps (green) and grey matter 3D maps (red). Centre of the figure: Functional domains of the corresponding RSNs. The functional domains' 3D maps are the union of the associated RSNs. Labelling indicates an arbitrary RSN number (in blue), the primary cortical anatomical landmarks (in black) and putative cognitive function (in orange). Ant. Sup. Par.: Anterior superior parietal network; Inf. central - SM head: Inferior central network (somato-motor, head portion); Lat. Occ.: Lateral occipital network; Lat. Post. Occ.: Lateral posterior occipital network; Med. Occ.: Medial occipital network; Med. Post. Occ.: Medial posterior occipital network; Mid. central (L) - SM hand (R): Middle central network, left hemisphere component (somatomotor, right hand portion); Mid. central (R) - SM hand (L): Middle central network, right 
hemisphere component (somato-motor, left hand portion); Post. Occ.: Posterior occipital network; Post. Sup. Par.: Posterior superior parietal network; Sup. central - SM body: Superior central network (somato-motor, body portion); Sup. Temp: Superior temporal network.

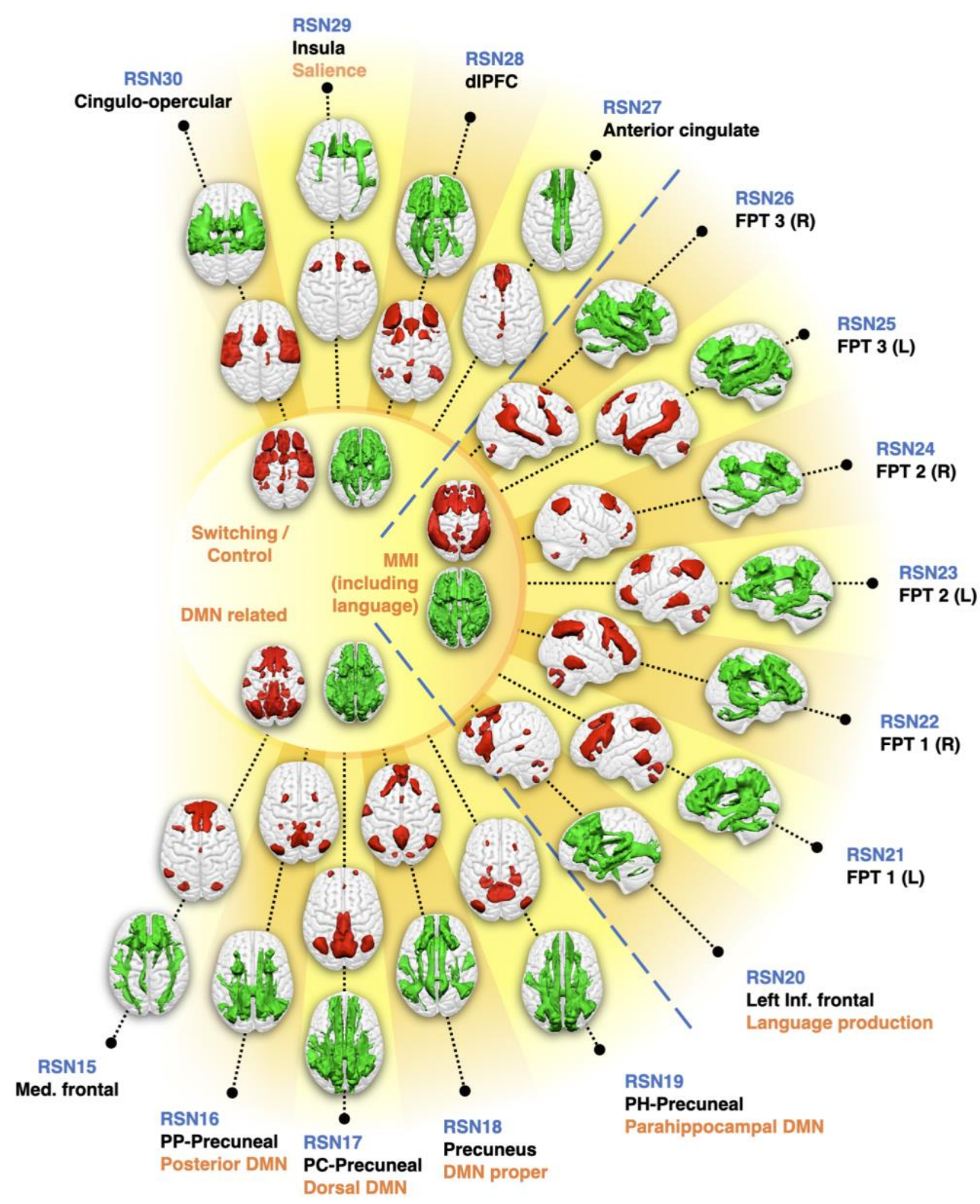

Figure 2: WhiteRest resting-state atlas of the switching/control, manipulation and maintenance of information (MMI), and default mode network (DMN) related domains. This composite figure shows the white matter 3D maps (green) and grey matter 3D maps (red). Centre of the figure: Functional domains of the corresponding RSNs. Labelling indicates an arbitrary RSN number (in blue), the primary cortical anatomical landmarks (in black) and putative cognitive function (in orange). DMN: Default Mode Network. dlPFC: 
Dorso-lateral prefrontal cortex network; FPT 1/2/3 (L/R): Fronto-parieto-temporal network 1/2/3, Left/Right hemisphere component; Med. frontal: Medial frontal network; PC-Precuneal: Posterior cingulate-precuneal network; PH-Precuneal: ParahippocampalPrecuneal network; PP-Precuneal: Posterior parietal-precuneal network.

The paired white matter and grey matter z-maps generated by our method were thresholded using an arbitrarily high threshold of 7 to get a highly conservative estimate of the RSNs' spatial extent. Using this threshold, the combined white matter maps cover $96 \%$ of the brain white matter, except for some orbito-frontal and ventro-temporal pathways, part of the internal capsule and part of the brain stem. Similarly, the combined grey matter maps cover $79 \%$ of the cortical grey matter, except for ventral areas in the temporal and frontal lobes.

The WhiteRest atlas reveals both the functional grey matter of an RSN and the structural white matter circuitry of this network. In the WhiteRest atlas, 21 of the 30 RSNs display a symmetrical pattern between the left and the right hemispheres. Eight networks are strongly lateralised, but with a contralateral homotopic counterpart, and one network was exclusively left lateralized (RSN20, language production network). To help further explore each RSN, a description of the maps of all the RSNs can be found in the supplementary material (Supplementary figures 1 - 30; the continuous maps are also available at https://neurovault.org/collections/AFVTMGXJ/ for the white matter, and https://neurovault.org/collections/SBZVMFMK/for the grey matter). As an illustrative example, the Default Mode Network (DMN) maps are showcased in Figure 3. Although the DMN can be described as a set of sub-networks, one of them is most representative of what is usually called "DMN" in the literature $(24,26,34)$ : the RSN18, which we labelled as "DMN proper". 


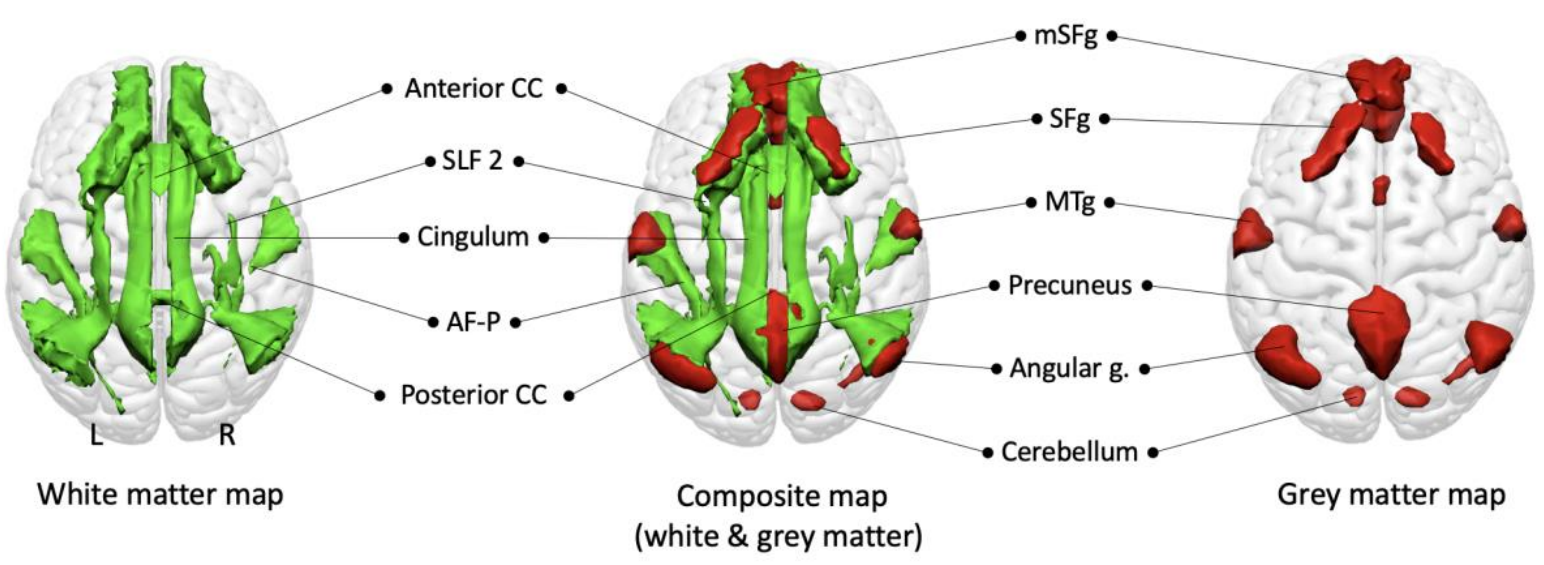

Figure 3: Default Mode Network proper (RSN18) maps, dorsal view. White matter map in green, grey matter map in red. Composite map in the middle. The cerebellum is visible through the glass-brain effect. AF-P: Arcuate fasciculus (posterior segment); mSFg.: medial superior frontal gyrus; MTg: Middle temporal gyrus; SFg: Superior frontal gyrus; SLF2: Second branch of the superior longitudinal fasciculus.

The grey matter map of the DMN proper revealed the bilateral involvement of the medial frontal cortex (the medial superior frontal gyrus, the gyrus rectus, and frontal pole), the superior frontal gyrus, the middle temporal gyrus, the precuneus, the angular gyrus and the cerebellum. The white matter maps of the RSN showed previously described pathways of the DMN, such as the second branch of the superior longitudinal fasciculus (SLF2) connecting the superior parietal lobe to the superior frontal gyrus and the cingulum connecting the precuneus area to the medial frontal area. Additionally, the middle temporal gyrus and the angular gyrus are connected by the posterior segment of the arcuate fasciculus. Interhemispheric connections were also present within the anterior and posterior corpus callosum connecting both frontal lobes and both precunei, respectively.

While the description of a known RSN, such as the DMN, can be used to validate the atlas, WhiteRest can also explore the uncharted white matter anatomy of RSNs, for instance, the Dorsal Attention Network (RSN13) presented in Figure 4. 


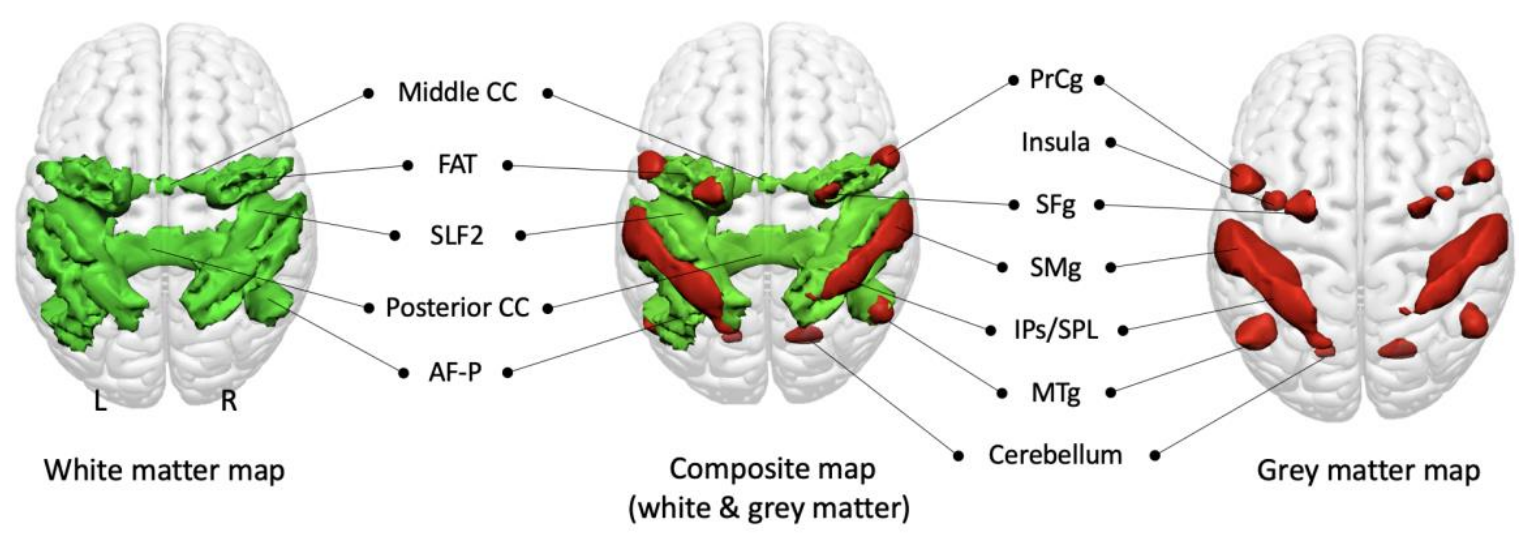

Figure 4: Dorsal Attention Network (RSN13) maps, dorsal view. White matter map in green, grey matter map in red. Union of the two maps in the middle. The insula and cerebellum are visible through the glass-brain effect. AF-P: Arcuate fasciculus (posterior segment); FAT: Frontal Aslant Tract; IPs/SPL: Intraparietal sulcus and superior parietal lobule; Middle CC: Middle part of the corpus callosum; MTg: Middle temporal gyrus; Posterior CC: Posterior part of the corpus callosum; PrCg: Precentral gyrus; Precentral s.: Precentral sulcus; SFg: Superior frontal gyrus; SLF2: Second branch of the Superior Longitudinal Fasciculus; SMg: Supramarginal gyrus.

The grey matter map revealed the involvement of core regions of the DAN, with the parietal cortex - supramarginal gyrus (SMg), intraparietal sulcus (IPs) and superior parietal lobule (IPL) - and part of the superior frontal gyrus (SMg), in the frontal eye field region. It also showed other areas associated with the DAN, namely the precentral gyrus (PrCg), the insula and the posterior part of middle temporal gyrus (MTg). The white matter map unveiled the involvement of the second branch of the superior longitudinal fasciculus (SLF2), connecting the inferior parietal cortex (IPs, SMg) with the frontal regions of the network (i.e. SFg, $\mathrm{PrCg}$ and insula). SFg and $\mathrm{PrCg}$ were also interconnected via the frontal aslant tract. The map also showed the involvement of the posterior segment of the arcuate fasciculus, connecting the MTg with the parietal cortex. Additionally, the map revealed the involvement of the corpus callosum, ensuring interhemispheric connectivity. 


\section{The overlapping nature of white matter RSNs}

The WhiteRest atlas suggests that most RSNs share white matter pathways with other RSNs. Indeed, most of the brain's white matter (i.e. 89\%) is shared amongst multiple RSN, with $16 \%$ of the white matter shared by at least 7 RSNs. By comparison, the grey matter contribution to RSNs show much less overlap, where $53 \%$ of the grey matter uniquely contribute to one RSN, and $45 \%$ to 2 or 3 RSNs. To determine the exact extent of the overlaps in the white matter, we generated an overlap map displaying the number of RSN per voxel in the brain. Large areas of the deep white matter showed high RSN overlap count (> 7 overlapping RSNs), including in the centrum semiovale and subportions of the medial corpus callosum (Fig. 5). RSNs also overlapped highly in the cingulum, the second and third branches of the superior longitudinal fasciculi (SLF2, SLF3), the arcuate fasciculi, and the inferior fronto-occipital fasciculi (IFOF) in both hemispheres. In contrast, the superficial white matter demonstrated less RSN overlap.

A

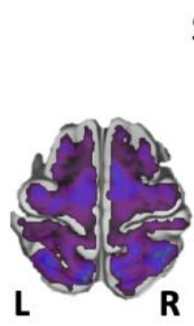

65
SLF2

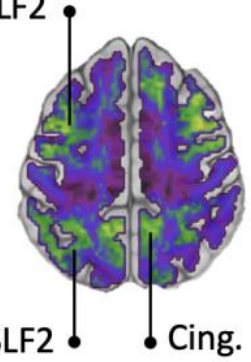

50

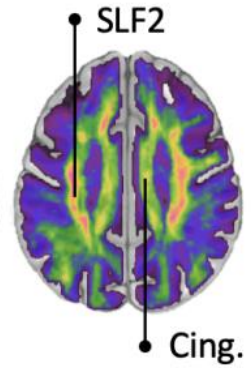

35

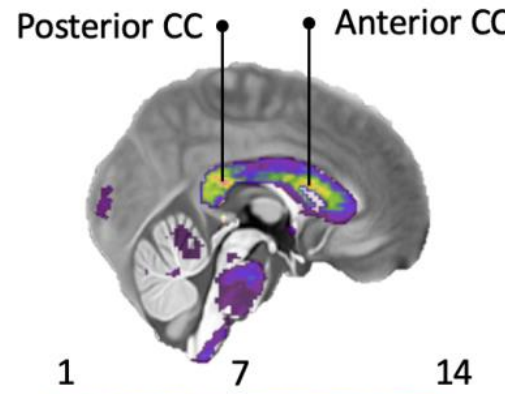

14
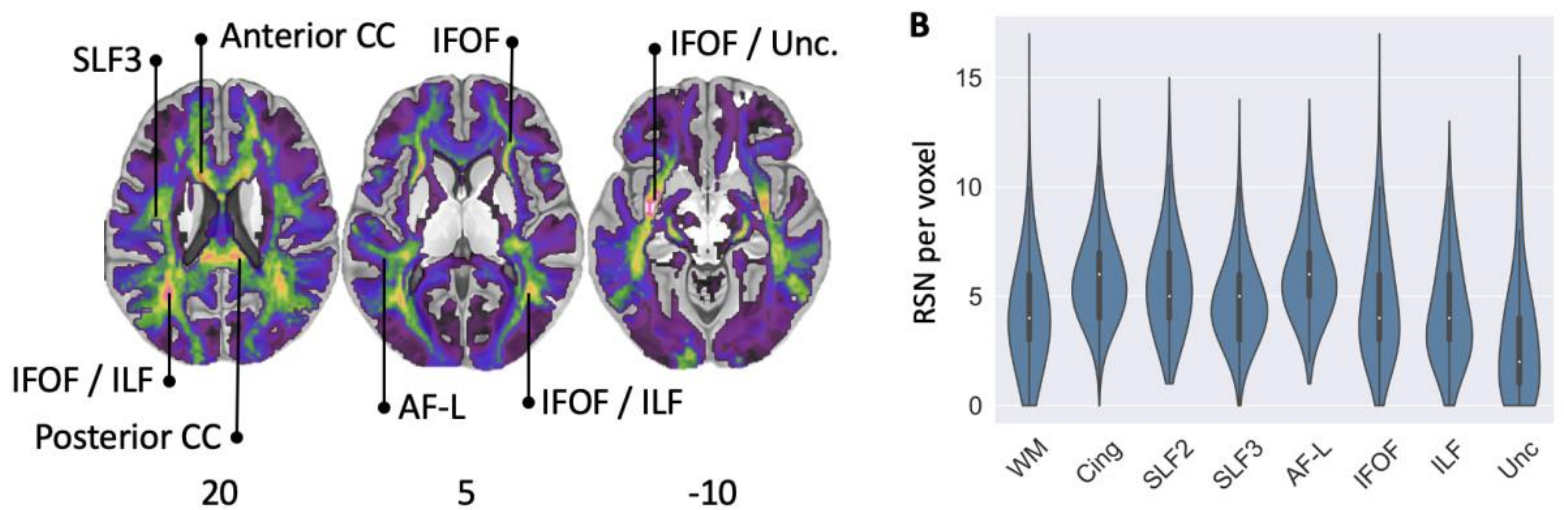

Figure 5: RSN overlap in the brain. A: Overlay map of RSN white matter maps. Colour bar: Number of RSN per voxel (saturated for $\mathrm{n}>14$ ). Anterior CC: Anterior corpus callosum; AFL: Arcuate fasciculus (long segment); Cing.: Cingulum; IFOF: Inferior fronto-occipital 
fasciculus; ILF: Inferior longitudinal fasciculus; Posterior CC: Posterior corpus callosum; SLF2: Second branch of the superior longitudinal fasciculus; SLF3: Third branch of the superior longitudinal fasciculus; Unc: Uncinate fasciculus. B: Violin plots (normalised by plotted area) of the overlap values in the total white matter and along the studied pathways (left and right hemispheres combined). WM: average whole white matter.

The existence of areas with high density RSN overlap in the white matter point toward the idea that lesions to the white matter could severely impact the functioning of multiple RSNs and hence cause a diverse pattern of clinical symptoms. To explore this aspect, we developed a new module, WhiteRest, freely available online through the Functionnectome software (available at http://toolkit.bcblab.com). WhiteRest can measure the involvement of each RSNs for any given region of interest (ROI) in the white matter or, more specifically, a given white matter lesion.

As an example, we investigated the RSNs potentially affected by a synthetic brain lesion (35) using the WhiteRest module (Fig. 6). The synthetic brain lesion included part of the centrum semiovale (CSO) in the left hemisphere (Fig. 6A), one of the areas with the highest overlap. The CSO is defined as a white matter area located superior to the lateral ventricle and characterised by the presence of projection, association, and commissural fibers (36). Here, the ROI of the synthetic lesion was defined as the intersection of the SLF2, the callosal fibers, and the cortico-spinal tract. The WhiteRest module outputs a table with the estimation of each involved RSN (Supplementary Table 1), as well as a pie-chart summarizing the results (Fig. 6B). 


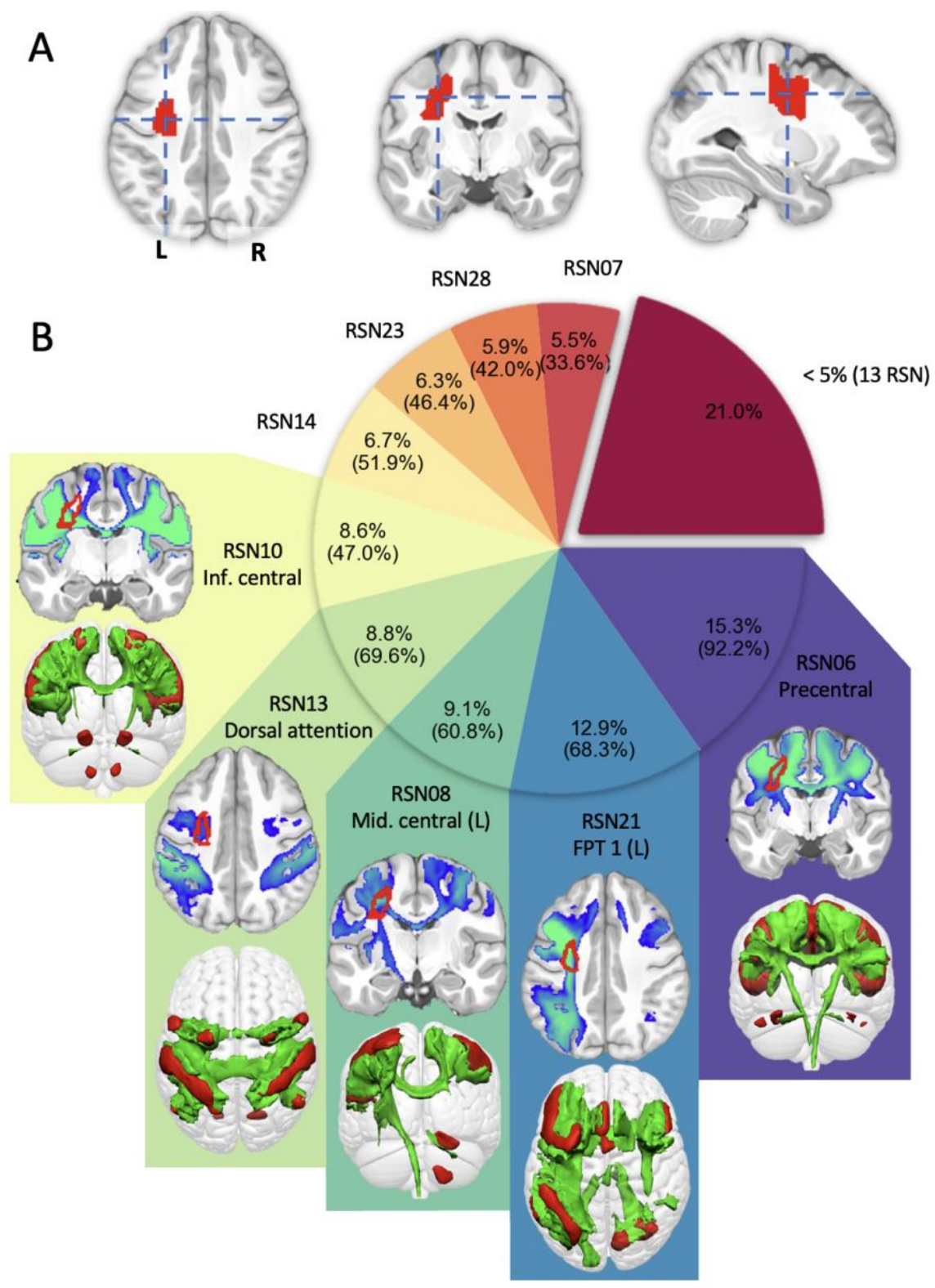

Figure 6: Participation of RSNs in the left centrum semiovale. A: Centrum semiovale ROI. B: RSN passing through the ROI, with relative presence (in \%) for each RSN, based on the z-value of the RSN maps, and fraction of the ROI covered by the RSN (between brackets). The 13 RSNs with less than 5\% participation are grouped together. The white matter maps of 5 most involved RSN are displayed, with a representative slice on top (ROI outlined in red), and the 3D map of white matter (in green) and grey matter (in red) underneath. Inf. central: Inferior central network; Mid. central (L): Middle central network (left hemisphere component); FPT 1 (L): Fronto-parieto-temporal network 1, left hemisphere component. 
As expected from such a fiber-crossing area, many (22 in total) RSNs were shown to be passing through the ROI. The 5 most involved RSNs were jointly responsible for more than half (54.7\%) of the total presence, while the 13 RSNs with low presence (less than 5\% each) amounted to 21\% of the total presence. The group of 5 RSNs with high presence was composed of central and precentral networks (RSN 06, 08 \& 10), as well as the DAN and the left FPT 1 network (RSN 13 \& 21). These networks can be associated with cerebral functions: motor functions with the central and precentral networks, attention with the DAN (37), and executive control with FPT 1 (38). This example illustrates how WhiteRest can be used to explore how lesions impact on specific RSNs. WhitRest, as part of the Functionnectome, will help to link lesions, RSN disruption, and clinical symptomes.

\section{Discussion}

We introduce WhiteRest, an atlas derived from integrated functional signal and structural information revealing white matter and grey matter components for each resting-state network. As such, the present work showcases two original results. First, the atlas itself, which consists of the systematic mapping of white matter contributing to the resting-state networks. Second, our results demonstrate that white matter pathways can contribute to multiple RSNs. This new atlas opens up the prospect of exploring the impact of white matter lesions on the integrity of resting-state networks and thus their functioning.

The WhiteRest atlas is, to our knowledge, the first comprehensive statistical mapping of the white matter contribution to RSNs. We generated white and grey matter maps concurrently, yielding continuous statistical maps of the RSNs in both tissues, thus allowing for a thorough exploration of each network. The combination of functional and structural information in our method can help the exhaustive detection of RSNs as there is evidence that structural connectivity holds complementary information regarding RSNs (29). Hence, the multimodality of the signal might help identifying and segregating networks as previously demonstrated by other groups with different modalities (e.g. Glasser's multi-modal parcellation (39)). Other attempts also combined grey matter functional and white matter structural information to explore the white matter 
contribution to subtending resting-states networks, but have been limited to a very low number of RSNs $(25,27)$. In contrast, recent works that undertook the atlasing of the RSN white matter connectivity did not directly combine functional and structural information. They mapped the RSN white matter circuits by connecting RSNs cortical regions from a pre-existing cortical RSNs atlas, using tractography data $(28,40)$. In this approach, the functional-structural mapping is highly dependent on the original cortical RSN atlas, while in our method both grey and white matter information are used concurrently.

Our data-driven method allowed for a global approach by mapping the whole brain, with the exception of ventral areas in zones strongly affected by magnetic susceptibility artefacts, where both the fMRI and diffusion signals are degraded (41). The individualICA-based scheme used to produce the statistical group maps revealed a fine granularity of the RSNs, where brain regions that are spatially distant but functionally and structurally connected are attributed to the same RSN. The fine granularity of the default mode network (DMN) in the WhiteRest atlas is a good example of the multimodal improvement of the networks' segregation. Our analysis replicated 4 previously described (42) DMN-related RSNs involving the precuneus (RSN 16, 17, 18 \& 19), while also differentiating a DMN proper (RSN18) from a medial frontal network (RSN15). For the DMN proper, the structural connectivity is largely known $(22-28,43)$, which offers a good opportunity to validate our method. For instance, WhiteRest's DMN proper white matter map confirmed the involvement of the cingulum, connecting the precuneus with the frontal cortex $(22-28,43)$, and of the superior longitudinal fasciculus (SLF2) connecting the superior frontal gyrus with the angular gyrus $(24,25,27)$. Similarly, the posterior segment of the arcuate fasciculus that connects the inferior parietal lobule with the posterior temporal lobe has been also reported in previous studies for the DMN $(22,24)$. Complementing the DMN-proper, in accordance with previous DMN descriptions (24), the medial frontal network involved the inferior longitudinal fasciculus (ILF, connecting the occipital lobe with the temporal lobe), the uncinate (connecting the temporal pole to the inferior frontal lobe), and the cingulum (connecting temporal-parietal-frontal areas). Similarly, the WhiteRest atlas can be used in a prospective and explorative manner, as shown with the unveiling of the dorsal attention network (RSN13). While the grey matter architecture of the DAN is well documented $(37,44)$, its white matter support has only been partially explored (45). To our knowledge, WhiteRest reveals the first 
comprehensive description of the DAN's white matter, that includes bilateral association fibres connecting ipsilateral regions, and commissural fibers ensuring interhemispheric connectivity. However, disentangling the exact functional relevance of each connection remains a challenge that will require, for example, functionnectome investigation (30) or advanced lesions analyses $(35,46,47)$. Such approaches might shed light on the hierarchical and functional implications of RSN circuits (46-49). Recent results have highlighted the importance of white matter structural disconnections in the disruption of functional connectivity (49), and this disruption has been linked to behavioural and cognitive dysfunction $(50,51)$. Therefore, being able to identify these RSN white matter "highways" would propel our understanding of disconnection symptoms, improve recovery prognostics, and inform preoperative brain surgery planning (52). In order to facilitate these efforts, we released the WhiteRest module (as part of the Functionnectome) that quantifies the presence of RSNs in a specific region of the brain's white matter. The WhiteRest module was designed to accept regions of interest (e.g. from parcellations or lesion) in the MNI 152 space $\left(2 \times 2 \times 2 \mathrm{~mm}^{3}\right)$ and provide an estimate of the RSNs involved, or in the case of lesions, which RSNs would be impacted by a lesion in this region.

As an example for lesion applications, we exemplified the use of the WhiteRest module to explore the left centrum semiovale, an area with a high number of overlapping RSNs and by definition a crossing region for many white matter pathways. Here, the software revealed that the CSO ROI was primarily involved in RSNs related to motor functions, visual attention, and executive control. As mentioned above, in a clinical framework such ROI could delineate a lesion, and the results would then be used to infer the lesion's disconnection effect on RSNs and cognitive symptoms. While the effects on RSNs of a CSO lesion are mostly unexplored, white matter damage has been linked to multiple dysfunctions (53), including motor (54) and executive $(55,56)$ impairments. These dysfunctions appear to correlate with cognitive functions of the RSNs identified with WhiteRest, which support its potential use as a tool for linking white matter damage with RSNs and brain functions. Overall, the WhiteRest module provides an opportunity for clinical neuroimagers to take advantage of an integrated functional and structural connectivity approach to shed light on the functional mechanisms of the brain and the origins of cognitive disorders. 
While the WhiteRest module and atlas represent an advance in the field of resting state functional neuroimaging, it is not exempt from limitations. For instance, we excluded the cerebellum-centered RSN in the present work. This decision was motivated by some limitations of tractography that are exacerbated in the cerebellum (57), mitigating the quality of the modeled pathways. For example, the fine structure of the cerebellum and the gathering of fibres in the brainstem are affected by partial volume and bottleneck effects (58). Also, some of the maps were displaying white matter pathways leading to grey matter areas absent on the related grey matter map. Some of these cases can be explained as simply threshold-dependent (i.e. $\mathrm{z}>7$ to facilitate the visualisation of 3D structures), which hid some of the less significant (but still involved) areas. However, these pathways might potentially correspond to the structural link between different RSNs. Thus, when exploring a network in detail, we strongly advise checking the nonthresholded maps as well to better appreciate the full white matter network involved in RSNs.

All in all, we introduced a novel combined atlas of resting-state networks, based on functional and structural connectivity to deliver white matter and grey matter maps for each RSN - the WhiteRest atlas. This atlas allows for the exploration of the structural support of individual RSN and facilitates the study of the impact of white matter lesions on resting-state networks. Accordingly, we released the WhiteRest module that estimates the proportion of RSNs impacted by a given white matter lesion. With this tool, future research can focus on exploring the link between white matter lesions and their effects on the related resting-state networks in light of symptom diagnosis. Leveraging a deep-learning approach recently introduced (42) opens up the possibility for individual resting-state functionnectome analyses and will facilitate a more personalised neuromedicine.

\section{Methods}

\section{HCP dataset}

The dataset used in the present study is composed of the openly-available resting-state scans (rsfMRI) from 150 participants (75 females; age range 22-35 years) of the Human 
Connectome Project (HCP) (32), with 45 participants from the test-rest HCP dataset and 105 randomly sampled participants from the Young adult dataset (http://www.humanconnectome.org/study/hcp-young-adult/; WU-Minn Consortium; Principal investigators: David Van Essen and Kamil Ugurbil; 1U54MH091657).

\section{Acquisition parameters}

\section{Preprocessing}

The resting-state acquisitions were then preprocessed using the "Minimal preprocessing pipeline" fMRIVolume (59), applying movement and distortion corrections, and registration to the MNI152 space. Further processing steps were also applied: despiking; voxelwise detrending of potentially unwanted signal (6 motion regressors, 18 motionderived regressors (60), and CSF, white matter, and grey matter mean time-courses); temporal filtering (0.01-0.1 Hz); and spatial smoothing (5 mm FWHM). While of exceptional quality, we chose to alter the HCP data to make it clinically relevant. A composite resting-state 4D volume was generated by discarding the 300 first and 300 last frames of the resting state acquisitions and concatenating (along the time axis) the resulting volumes. For each subject this corresponded to 7.5 minutes with the left-right and 7.5 minutes with the right-left phase of acquisition (= 1200 frames total).

\section{Extraction of white matter and grey matter components}

\section{Projection of the functional signal to the white matter}

In order to explore the white matter structures of resting-state networks, we projected the functional signal from the rs-fMRI scans onto the white matter using the Functionnectome (30, 31) (https://github.com/NotaCS/Functionnectome). The Functionnectome is a recently introduced method that unlocks the functional study of the white matter. Briefly, the Functionnectome takes an fMRI scan and a grey matter mask as inputs, combines grey matter BOLD signal with white matter anatomical priors, and outputs a new functional volume (called a functionnectome), with the same 
dimensions as the original fMRI scan (same 3D space, same number of time-points), but with the functional signal associated to the white matter.

In this functionnectome volume, the signal of a white matter voxel results from the combination of the BOLD signals from the voxels within the grey matter mask that are structurally connected to it (weighted by the probability of connection). The structural connectivity probability is given by the anatomical priors provided with software (customisable priors option available). Using the Functionnectome thus allows the analysis of the functional signal in a connectivity-oriented framework by integrating the signal from distant but structurally connected grey matter voxels or clusters of voxels.

For our analysis, each of the 150 rs-fMRI scans from the dataset were processed with the Functionnectome, along with a grey matter mask (the same mask for all the subjects). This mask was computed using the brain parcellation data from all the participants: the mask corresponds to the voxels that were identified as part of the grey matter in at least $10 \%$ of the participants. This processing produced 150 resting-state functionnectome (rsfunctionnectome) volumes, one per participant.

\section{Independent component analysis}

To extract RSN from the data, we used an independent component analysis (ICA) method. For each subject, the original rs-fMRI scan was spatially concatenated with the associated rs-functionnectome. This resulted in functional volumes containing, side by side, the original resting-state signal (on the grey matter) and the rs-functionnectome signal (on the white matter). These composite functional volumes were then analysed with MELODIC (multivariate exploratory linear optimised decomposition into independent components, version 3.15), from the FMRIB Software Library (FSL) (61) to extract independent components (ICs) from the signal. The number of IC per participant was individually determined by Laplace approximation (62). This resulted in a set of ICs, unlabeled putative RSNs, per participant. Each IC was composed of a temporal component (the IC's time-course) and a spatial map, displaying side by side (due to the above-mentioned spatial concatenation) the component in rs-fMRI (i.e. grey matter) space and in the rs-functionnectome (i.e. white matter) space. Each IC was then split into paired white matter maps and grey matter maps. 


\section{Generating RSN maps by clustering ICs}

We used MICCA (33), an unsupervised classification algorithm developed to automatically group ICs from multiple individual ICAs (from different participants) based on the similarity of their spatial maps. The resulting groups, composed of ICs reproducible across participants, were used to produce group maps. Such an individualbased ICA scheme was preferred to the classical group ICA as some evidence suggests that group ICA can fail to separate some RSNs if they share functional hubs (33).

The atlas was produced by applying MICCA using the procedure described in the original Naveau et al. paper (33), in a 5 repetition scheme (i.e. ICA and MICCA were repeated 5 times per participant, and the resulting IC groups were paired with ICASSO (63)). The procedure generated 36 IC groups and their associated z-map, reproducible across the repetitions. Among them, 5 groups were identified as artefacts and were excluded, and 1 was located in the cerebellum and was excluded too in later analyses. We thus obtained a total of 30 RSNs, producing the WhiteRest atlas. Each RSN was then named by experts (MJ, VN) according to its anatomical localisation and in reference to AAL $(64,65)$ and AICHA $(64)$ atlas. Likewise, the classification of RSNs to a functional domain was done by an expert using the grey matter spatial patterns and estimated functional role of the RSNs presented here, compared to the one from Doucet et al. (12)

Note that we applied MICCA on the grey matter maps of the ICs. We used only these maps for the clustering as MICCA has been developed and validated to cluster only classical resting-state derived spatial maps (in grey matter space). As each grey matter map is associated to a white matter map (since they are part of the same IC), the procedure still produces paired grey an white matter RSN maps, as presented in the atlas.

\section{Overlap analysis}

To measure the extent of overlaps between RSNs in the white matter, all the maps were thresholded ( $\mathrm{z}>7)$, binarized, and summed, generating a new map with the number of RSN per voxel.

Additionally, we provided a new software, WhiteRest, to explore how the white matter is shared between RSNs. Given an ROI in the white matter, it measures the "proportional

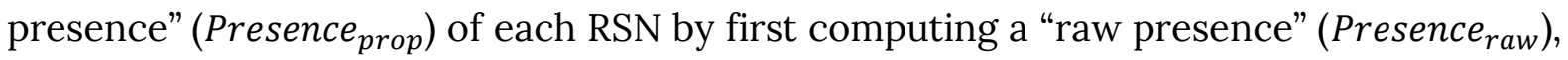
adding up the $\mathrm{z}$-score value of all the RSN white matter map voxels in the ROI (Equation 
1). This raw presence score is then converted into a proportional presence by dividing it by the sum of the raw presence score of all the RSNs sharing part of the ROI (Equation 2): For a given RSN and a given ROI,

$$
\begin{gathered}
\text { Presence }_{\text {raw }}(R S N, R O I)=\sum_{v \in R O I} Z_{R S N}(v) \\
\text { Presence }_{\text {prop }}(R S N, R O I)=\frac{\text { Presence }_{\text {raw }}(R S N, R O I)}{\sum_{\text {rsn } \in \text { Atlas } \text { Presence }_{\text {raw }}(r S n, R O I)}}
\end{gathered}
$$

with $v$ a voxel from the ROI, $z_{\mathrm{RSN}}(v)$ the $\mathrm{z}$-score of the voxel $v$ in the white matter map of the studied RSN.

Note that the WhiteRest module outputs both raw and proportional presence scores.

To demonstrate the results obtained with WhiteRest, we used a ROI delimiting the centrum semiovale as input. This ROI was generated using the Atlas of Human Brain Connections (66), available at http://www.bcblab.com/BCB/Opendata.html, and was defined as the intersection of the second branch of the superior longitudinal fasciculus, the cortico-spinal tract, and the callosal fibers, with each maps from the atlas thresholded at 0.5 .

\section{Visualisation}

The 3D z-maps presented in figure $1,2,3,4 \& 5$ were generated using Surf Ice (https://www.nitrc.org/projects/surfice/), with the default mni152_2009 brain volume as background template. The 2D brain slices of figure $5 \& 6$ were displayed on a standard template in MRIcron (https://www.nitrc.org/projects/mricron). Each white matter map was masked in order to remove the grey matter part of the volume to improve readability. The mask used corresponded to voxels defined as white matter in at least $10 \%$ of the 150 participants, according to the parcellation provided with the HCP datasets. In figure 5, the RSN count was saturated at 14 on the displayed map to improve readability, as only a handful of voxels presented higher values.

\section{Acknowledgments}

We thank the University of Bordeaux and CNRS for the infrastructural support. This project has received funding from the European Research Council (ERC) under the European Union's Horizon 2020 research and innovation programme (grant agreement 
No. 818521; MTS) and the Marie Skłodowska-Curie programme (grant agreement No. 101028551; SJF).

Part of the computing was done thanks to the computing facilities MCIA (Mésocentre de Calcul Intensif Aquitain) of the University of Bordeaux and of the Universite de Pau et des Pays de l'Adour.

Data were provided by the Human Connectome Project, WU-Minn Consortium (Principal Investigators: David Van Essen and Kamil Ugurbil; 1U54MH091657) funded by the 16 NIH Institutes and Centers that support the NIH Blueprint for Neuroscience Research; and by the McDonnell Center for Systems Neuroscience at Washington University.

\section{Data availability}

The WhiteRest atlas (non thresholded maps) is freely available on Neurovault.org, with both the grey matter maps (https://neurovault.org/collections/SBZVMFMK/) and the white matter maps (https://neurovault.org/collections/AFVTMGXJ/).

The WhiteRest module is open-source and freely available as part of the Functionnectome software, which can be found at http://www.bcblab.com/BCB/Opendata.html or directly downloaded from https://github.com/NotaCS/Functionnectome.

\section{References}

1. S. Ogawa, T. M. Lee, A. R. Kay, D. W. Tank, Brain magnetic resonance imaging with contrast dependent on blood oxygenation. Proc. Natl. Acad. Sci. U. S. A. 87, 9868$9872(1990)$.

2. S. M. Smith, et al., Correspondence of the brain's functional architecture during activation and rest. Proc. Natl. Acad. Sci. U. S. A. 106, 13040-13045 (2009).

3. B. Biswal, F. Z. Yetkin, V. M. Haughton, J. S. Hyde, Functional connectivity in the motor cortex of resting human brain using echo-planar MRI. Magn. Reson. Med. 34, 537-541 (1995).

4. M. D. Fox, et al., The human brain is intrinsically organized into dynamic, anticorrelated functional networks. Proc. Natl. Acad. Sci. U. S. A. 102, 9673-9678 (2005).

5. C. F. Beckmann, M. DeLuca, J. T. Devlin, S. M. Smith, Investigations into resting- 
state connectivity using independent component analysis. Philos. Trans. R. Soc. Lond. B Biol. Sci. 360, 1001-1013 (2005).

6. J. S. Damoiseaux, et al., Consistent resting-state networks across healthy subjects. Proc. Natl. Acad. Sci. U. S. A. 103, 13848-13853 (2006).

7. F. Agosta, et al., Resting state fMRI in Alzheimer's disease: beyond the default mode network. Neurobiol. Aging 33, 1564-1578 (2012).

8. B. Mohammadi, et al., Changes of resting state brain networks in amyotrophic lateral sclerosis. Exp. Neurol. 217, 147-153 (2009).

9. F. Orliac, et al., Links among resting-state default-mode network, salience network, and symptomatology in schizophrenia. Schizophr. Res. 148, 74-80 (2013).

10. M. H. Lee, C. D. Smyser, J. S. Shimony, Resting-state fMRI: a review of methods and clinical applications. AJNR Am. J. Neuroradiol. 34, 1866-1872 (2013).

11. M. P. van den Heuvel, H. E. Hulshoff Pol, Exploring the brain network: a review on resting-state fMRI functional connectivity. Eur. Neuropsychopharmacol. 20, 519-534 (2010).

12. G. Doucet, et al., Brain activity at rest: a multiscale hierarchical functional organization. J. Neurophysiol. 105, 2753-2763 (2011).

13. V. D. Calhoun, K. A. Kiehl, G. D. Pearlson, Modulation of temporally coherent brain networks estimated using ICA at rest and during cognitive tasks. Hum. Brain Mapp. 29, 828-838 (2008).

14. C. Jutten, J. Herault, Blind separation of sources, part I: An adaptive algorithm based on neuromimetic architecture. Signal Processing 24, 1-10 (1991).

15. E. O. Stejskal, J. E. Tanner, Spin diffusion measurements: Spin echoes in the presence of a time-dependent field gradient. J. Chem. Phys. 42, 288-292 (1965).

16. B. Jeurissen, M. Descoteaux, S. Mori, A. Leemans, Diffusion MRI fiber tractography of the brain. NMR Biomed. 32, e3785 (2019).

17. M. Catani, M. Thiebaut de Schotten, Atlas of Human Brain Connections (2012) https:/doi.org/10.1093/med/9780199541164.001.0001.

18. G. J. M. Parker, H. A. Haroon, C. A. M. Wheeler-Kingshott, A framework for a streamline-based probabilistic index of connectivity (PICo) using a structural interpretation of MRI diffusion measurements. J. Magn. Reson. Imaging 18, 242-254 (2003).

19. G. Girard, K. Whittingstall, R. Deriche, M. Descoteaux, Towards quantitative connectivity analysis: reducing tractography biases. Neuroimage 98, 266-278 (2014).

20. A. M. Hermundstad, et al., Structural foundations of resting-state and task-based functional connectivity in the human brain. Proc. Natl. Acad. Sci. U. S. A. 110, 61696174 (2013). 
21. C. J. Honey, et al., Predicting human resting-state functional connectivity from structural connectivity. Proc. Natl. Acad. Sci. U. S. A. 106, 2035-2040 (2009).

22. M. D. Greicius, K. Supekar, V. Menon, R. F. Dougherty, Resting-state functional connectivity reflects structural connectivity in the default mode network. Cereb. Cortex 19, 72-78 (2009).

23. S. Khalsa, S. D. Mayhew, M. Chechlacz, M. Bagary, A. P. Bagshaw, The structural and functional connectivity of the posterior cingulate cortex: comparison between deterministic and probabilistic tractography for the investigation of structurefunction relationships. Neuroimage 102 Pt 1, 118-127 (2014).

24. P. N. Alves, et al., An improved neuroanatomical model of the default-mode network reconciles previous neuroimaging and neuropathological findings. Commun Biol 2 , 370 (2019).

25. A. Tarun, H. Behjat, T. Bolton, D. Abramian, D. Van De Ville, Structural mediation of human brain activity revealed by white-matter interpolation of fMRI. Neuroimage 213, 116718 (2020).

26. M. P. van den Heuvel, R. C. W. Mandl, R. S. Kahn, H. E. Hulshoff Pol, Functionally linked resting-state networks reflect the underlying structural connectivity architecture of the human brain. Hum. Brain Mapp. 30, 3127-3141 (2009).

27. F. Calamante, et al., Track-weighted functional connectivity (TW-FC): a tool for characterizing the structural-functional connections in the brain. Neuroimage $\mathbf{7 0}$, 199-210 (2013).

28. T. D. Figley, N. Bhullar, S. M. Courtney, C. R. Figley, Probabilistic atlases of default mode, executive control and salience network white matter tracts: an fMRI-guided diffusion tensor imaging and tractography study. Front. Hum. Neurosci. 9, 585 (2015).

29. J. O'Muircheartaigh, S. Jbabdi, Concurrent white matter bundles and grey matter networks using independent component analysis. Neuroimage 170, 296-306 (2018).

30. V. Nozais, S. J. Forkel, C. Foulon, L. Petit, M. Thiebaut de Schotten, Functionnectome as a framework to analyse the contribution of brain circuits to fMRI. Commun Biol 4, 1035 (2021).

31. V. Nozais, NotaCS/Functionnectome: First official release (Zenodo, 2021) https:/doi.org/10.5281/ZENODO.5078062.

32. D. C. Van Essen, et al., The WU-Minn Human Connectome Project: an overview. Neuroimage 80, 62-79 (2013).

33. M. Naveau, et al., A novel group ICA approach based on multi-scale individual component clustering. Application to a large sample of fMRI data. Neuroinformatics 10, 269-285 (2012).

34. M. E. Raichle, The brain's default mode network. Annu. Rev. Neurosci. 38, 433-447 
(2015).

35. M. Thiebaut de Schotten, C. Foulon, P. Nachev, Brain disconnections link structural connectivity with function and behaviour. Nat. Commun. 11, 5094 (2020).

36. F. Vicq d'Azyr, Traité d'anatomie et de physiologie (Didot l'Aine, 1786).

37. M. Corbetta, G. L. Shulman, Control of goal-directed and stimulus-driven attention in the brain. Nat. Rev. Neurosci. 3, 201-215 (2002).

38. M. L. Dixon, et al., Heterogeneity within the frontoparietal control network and its relationship to the default and dorsal attention networks. Proc. Natl. Acad. Sci. U. S. A. 115, E1598-E1607 (2018).

39. M. F. Glasser, et al., A multi-modal parcellation of human cerebral cortex. Nature 536, 171-178 (2016).

40. T. D. Figley, et al., Probabilistic White Matter Atlases of Human Auditory, Basal Ganglia, Language, Precuneus, Sensorimotor, Visual and Visuospatial Networks. Front. Hum. Neurosci. 11, 306 (2017).

41. J. G. Ojemann, et al., Anatomic localization and quantitative analysis of gradient refocused echo-planar fMRI susceptibility artifacts. Neuroimage 6, 156-167 (1997).

42. V. Nozais, et al., Deep Learning-based Classification of Resting-state fMRI Independent-component Analysis. Neuroinformatics (2021) https:/doi.org/10.1007/s12021-021-09514-x.

43. A. Horn, D. Ostwald, M. Reisert, F. Blankenburg, The structural-functional connectome and the default mode network of the human brain. Neuroimage $102 \mathbf{P t}$ 1, 142-151 (2014).

44. M. D. Fox, M. Corbetta, A. Z. Snyder, J. L. Vincent, M. E. Raichle, Spontaneous neuronal activity distinguishes human dorsal and ventral attention systems. Proc. Natl. Acad. Sci. U. S. A. 103, 10046-10051 (2006).

45. P. G. Allan, et al., Parcellation-based tractographic modeling of the dorsal attention network. Brain Behav. 9, e01365 (2019).

46. C. Foulon, et al., Advanced lesion symptom mapping analyses and implementation as BCBtoolkit. Gigascience 7, 1-17 (2018).

47. J. C. Griffis, N. V. Metcalf, M. Corbetta, G. L. Shulman, Damage to the shortest structural paths between brain regions is associated with disruptions of restingstate functional connectivity after stroke. Neuroimage 210, 116589 (2020).

48. C.-H. Park, et al., Longitudinal changes of resting-state functional connectivity during motor recovery after stroke. Stroke 42, 1357-1362 (2011).

49. J. C. Griffis, N. V. Metcalf, M. Corbetta, G. L. Shulman, Structural disconnections explain brain network dysfunction after stroke. Cell Rep. 28, 2527-2540.e9 (2019). 
50. S. Li, et al., Altered resting-state functional and white matter tract connectivity in stroke patients with dysphagia. Neurorehabil. Neural Repair 28, 260-272 (2014).

51. J.-R. Ding, et al., Altered connectivity patterns among resting state networks in patients with ischemic white matter lesions. Brain Imaging Behav. 12, 1239-1250 (2018).

52. P. Branco, et al., Resting-State Functional Magnetic Resonance Imaging for Language Preoperative Planning. Front. Hum. Neurosci. 10, 11 (2016).

53. S. J. Read, et al., White matter medullary infarcts: acute subcortical infarction in the centrum ovale. Cerebrovasc. Dis. 8, 289-295 (1998).

54. M. Dinomais, et al., Long term motor function after neonatal stroke: Lesion localization above all. Human Brain Mapping 36, 4793-4807 (2015).

55. R. Vataja, et al., MRI correlates of executive dysfunction in patients with ischaemic stroke. European Journal of Neurology 10, 625-631 (2003).

56. P. Kourtidou, et al., Centrum semiovale and corpus callosum integrity in relation to information processing speed in patients with severe traumatic brain injury. J. Head Trauma Rehabil. 28, 433-441 (2013).

57. M. Catani, From hodology to function. Brain 130, 602-605 (2007).

58. F. Rheault, P. Poulin, A. Valcourt Caron, E. St-Onge, M. Descoteaux, Common misconceptions, hidden biases and modern challenges of dMRI tractography. J. Neural Eng. 17, 011001 (2020).

59. M. F. Glasser, et al., The minimal preprocessing pipelines for the Human Connectome Project. Neuroimage 80, 105-124 (2013).

60. K. J. Friston, S. Williams, R. Howard, R. S. J. Frackowiak, R. Turner, MovementRelated effects in fMRI time-series. Magnetic Resonance in Medicine 35, 346-355 (1996).

61. S. M. Smith, et al., Advances in functional and structural MR image analysis and implementation as FSL. Neuroimage 23 Suppl 1, S208-19 (2004).

62. T. Minka, Automatic choice of dimensionality for PCA. Adv. Neural Inf. Process. Syst. 13, 598-604 (2000).

63. J. Himberg, A. Hyvärinen, F. Esposito, Validating the independent components of neuroimaging time series via clustering and visualization. Neuroimage 22, 1214-1222 (2004).

64. M. Joliot, et al., AICHA: An atlas of intrinsic connectivity of homotopic areas. J. Neurosci. Methods 254, 46-59 (2015).

65. N. Tzourio-Mazoyer, et al., Automated anatomical labeling of activations in SPM using a macroscopic anatomical parcellation of the MNI MRI single-subject brain. Neuroimage 15, 273-289 (2002). 
66. K. Rojkova, et al., Atlasing the frontal lobe connections and their variability due to age and education: a spherical deconvolution tractography study. Brain Struct. Funct. 221, 1751-1766 (2016). 